\title{
p-Riesz bases in quasi shift invariant spaces
}

\author{
Laura De Carli, Pierluigi Vellucci
}

\begin{abstract}
Let $1 \leq p<\infty$ and let $\psi \in L^{p}\left(\mathbb{R}^{d}\right)$. We study $p$-Riesz bases of quasi shift invariant spaces $V^{p}(\psi ; Y)$.
\end{abstract}

\section{Introduction}

Let $1 \leq p<\infty$ and let $\psi \in L^{p}\left(\mathbb{R}^{d}\right)$. We consider the shift invariant space $V^{p}(\psi)=\overline{\operatorname{Span}\left\{\tau_{k} \psi\right\}_{k \in \mathbb{Z}^{d}}}$, where $\tau_{s} f(x)=f(x+s)$ is the translation and "bar" denotes the closure in $L^{p}\left(\mathbb{R}^{d}\right)$. Shift-invariant spaces appear naturally in signal theory and in other branches of applied sciences. In [2] [13] and in the recent preprint [14] quasi-shift invariant spaces of functions are considered. Given $X=\left\{x_{j}\right\}_{j \in \mathbb{Z}^{d}}$, a countable and discrete $\mathbb{1}^{1}$ subset of $\mathbb{R}^{d}$ and a function $\psi \in L^{p}\left(\mathbb{R}^{d}\right)$, we let

$$
V^{p}(\psi ; X)=\overline{\operatorname{Span}\left\{\tau_{x_{j}} \psi\right\}}
$$

Thus, $V^{p}(\psi)=V^{p}\left(\psi ; \mathbb{Z}^{d}\right)$. Quasi-shift invariant spaces are also called Spline-type spaces in [9], [10] [1], 23].

Following [2], 7], we say that the translates $\left\{\tau_{x_{j}} \psi\right\}_{j \in \mathbb{Z}^{d}}$ form a p-Riesz basis in $V^{p}(\psi ; X)$ if there exist constants $A, B>0$ such that, for every finite set of coefficients $\vec{d}=\left\{d_{j}\right\} \subset \mathbb{C}$,

$$
A\|\vec{d}\|_{\ell^{p}} \leq\left\|\sum_{j} d_{j} \tau_{x_{j}} \psi\right\|_{p} \leq B\|\vec{d}\|_{\ell^{p}}
$$

Here and throughout the paper, we have let $\|f\|_{p}=\left(\int_{\mathbb{R}^{d}}|f(x)|^{p} d x\right)^{\frac{1}{p}}$ and $\|\vec{c}\|_{\ell^{p}}=\left(\sum_{j \in \mathbb{Z}^{d}}\left|c_{j}\right|^{p}\right)^{\frac{1}{p}}$. If $x=\left(x_{1}, \ldots, x_{d}\right), y=\left(y_{1}, \ldots, y_{d}\right) \in \mathbb{R}^{d}$, we will often let $x \cdot y=x_{1} y_{1}+\ldots+x_{d} y_{d}$ and $|x|_{2}=(x \cdot x)^{\frac{1}{2}}$. We will also let $|x|_{\infty}=\sup _{1 \leq j \leq d}\left|x_{j}\right|$.

\footnotetext{
${ }^{1}$ A countable set $X \subset \mathbb{R}^{d}$ is discrete if for every $x_{j} \in X$ there exists $\delta_{j}>0$ such that $\left|x_{j}-x_{k}\right|_{2}>\delta_{j}$ for every $k \neq j$.
} 
If (1.2) holds, then it is possible to prove that

$$
2 V^{p}(\psi ; X)=\left\{f=\sum_{k \in \mathbb{Z}^{d}} d_{k} \tau_{x_{k}} \psi(x), \vec{d} \in \ell^{p}\right\}
$$

and the sequence $\left\{d_{k}\right\}_{k \in \mathbb{Z}^{d}}$ is uniquely determined by $f$.

$p$-Riesz bases allow a stable reconstruction of functions in $V^{p}(\psi ; X)$; when $X=\mathbb{Z}^{d}$ and $\mathcal{B}=\left\{\tau_{j} \psi\right\}_{j \in \mathbb{Z}^{d}}$ is a $p$-Riesz basis of $V^{p}(\psi)$, the coefficient $d_{j}$ in (1.3) can be expressed in an unique way in terms of the functions in the dual basis of $\mathcal{B}$. See 30, 5 and also [1 for explicit reconstruction formulas.

When $\psi$ has compact support, it is known (see e.g. [1, Prop. 1.1], [16], [24]) that $\mathcal{B}$ is a $p$-Riesz basis in $V^{p}(\psi)$ if and only if $\sum_{m \in \mathbb{Z}^{d}}|\hat{\psi}(y+m)|^{2} \neq 0$ for every $y \in\left[-\frac{1}{2}, \frac{1}{2}\right)^{d}$ and every $m \in \mathbb{Z}^{d}$. See also Lemma 5 in Section 2.

We have denoted with $\hat{\psi}(y)=\int_{\mathbb{R}^{d}} e^{2 \pi i x \cdot y} f(x) d x$ the Fourier transform of $\psi$. The proof of the aforementioned result relies on the lattice structure of $\mathbb{Z}^{d}$ and on standard Fourier analysis technique and does not easily generalize to other sets of translations.

Let $\psi \in L^{p}\left(\mathbb{R}^{d}\right), 1 \leq p<\infty$, and let $X=\left\{x_{j}\right\}_{j \in \mathbb{Z}^{d}}$ be a discrete set of $\mathbb{R}^{d}$. It is natural to consider the following problem:

Problem 1. Let $\mathcal{B}_{X}=\left\{\tau_{x_{j}} \psi\right\}_{j \in \mathbb{Z}^{d}}$ be a p-Riesz basis for $V^{p}(\psi ; X)$; can we find $\delta>0$ so that, for every $Y=\left\{y_{j}\right\}_{j \in \mathbb{Z}^{d}} \subset \mathbb{R}^{d}$ with $\sup _{j}\left|y_{j}-x_{j}\right|_{2}<\delta$, the set $\mathcal{B}_{Y}=\left\{\tau_{y_{j}} \psi\right\}_{j \in \mathbb{Z}^{d}}$ is a $p$-Riesz basis for $V^{p}(\psi ; X)$ ?

This problem cannot be solved if $\psi$ has compact support. For example, let $\psi(x)=\operatorname{rect}(x)$ be the characteristic function of the interval $\left[-\frac{1}{2}, \frac{1}{2}\right)$ and let $X=\mathbb{Z}$; let $Y=\left\{y_{n}\right\}_{n \in \mathbb{Z}}$ be such that $y_{0}=\delta>0$ and $y_{n}=n$ when $n \neq 0$. All functions in $V^{p}($ rect; $Y)$ vanish in the interval $\left[-\frac{1}{2},-\frac{1}{2}+\delta\right]$ and so $V^{p}($ rect; $Y) \neq V^{p}$ (rect).

We prove in Section 3 that Problem 1 can be solved when $p=2$ and $\psi$ is band-limited, i.e., when the Fourier transform of $\psi$ has compact support. See also Section 5 for more remarks and comments on problem 1

We are concerned with the following problem:

Problem 2. With the notation of Problem 1: let $\mathcal{B}_{X}$ be a p-Riesz basis for $V^{p}(\psi ; X)$ and let $Y=\left\{y_{n}\right\}_{n \in \mathbb{Z}^{d}}$ that satisfies $\sup _{n}\left|y_{n}-x_{n}\right|_{2}<\delta$; is $\mathcal{B}_{Y}$ a p-Riesz basis for $V^{p}(\psi ; Y)$ whenever $\delta$ is sufficiently small?

It is proved in [10 that Problem 2 has always solution when $X$ is relatively separated, i.e., when $X=X_{1} \cup \ldots \cup X_{k}$, with $X_{j}=\left\{x_{j, n}\right\}_{n \in \mathbb{Z}^{d}}$ and $\inf _{n \neq m}\left|x_{j, n}-x_{j, m}\right|_{2}>0$.

In Section 2 we prove the following theorem.

Theorem 1. Suppose that that $\psi$ is in the Sobolev space $W^{1, p}\left(\mathbb{R}^{d}\right)$, with $1<p<\infty$, and that $\left\{\tau_{x_{j}} \psi\right\}_{j \in \mathbb{Z}^{d}}$ is a $p$-Riesz basis of $V^{p}(\psi ; X)$. For

\footnotetext{
${ }^{2}$ A proof of this ientity was kindly provided to us by K. Hamm
} 
every $j \in \mathbb{Z}^{d}$ there exists $\delta_{j}>0$ such that $\left\{\tau_{y_{j}} \psi\right\}_{j \in \mathbb{Z}^{d}}$ is a p-Riesz basis of $V^{p}(\psi ; Y)$ whenever $\left|x_{j}-y_{j}\right|_{2}<\delta_{j}$.

We recall that $W^{1, p}(D)$ is the space of $L^{p}(D)$ functions whose partial distributional derivatives are also in $L^{p}(D)$ and that $W_{0}^{1, p}(D)$ is the closure of $C_{0}^{\infty}(D)$ in $W^{1, p}(D)$.

When $X$ is not relatively separated the $\delta_{j}$ 's in Theorem 1 may not have a positive lower bound, but we can still solve Problem 2 in the cases considered in Theorems 2 and 3 below.

Theorem 2. Assume that $\psi \in L^{1}\left(\mathbb{R}^{d}\right) \cap L^{2}\left(\mathbb{R}^{d}\right)$ satisfies

$$
0<c=\sum_{k \in \mathbb{Z}^{d}} \inf _{x \in[0,1)^{d}}|\hat{\psi}(x+k)|^{2}<\sum_{k \in \mathbb{Z}^{d}} \sup _{x \in[0,1)^{d}}|\hat{\psi}(x+k)|^{2}=C<\infty .
$$

Then, Problem 2 can be solved when $p=2$ and $\left\{e^{2 \pi i x_{n} \cdot x}\right\}_{n \in \mathbb{Z}^{d}}$ is a Riesz basis in $L^{2}\left([0,1)^{d}\right)$.

We recall that the amalgam space $W\left(L^{\infty}, \ell^{q}\right)$ is the set of measurable functions $f: \mathbb{R}^{d} \rightarrow \mathbb{C}$ for which $\|f\|_{W\left(L^{\infty}, \ell^{q}\right)}=$ $\left(\sum_{n \in \mathbb{Z}^{d}} \sup _{x \in[0,1)^{d}}|f(x+n)|_{2}^{q}\right)^{\frac{1}{q}}<\infty$. The amalgam space $W\left(L^{r}, \ell^{q}\right)$ can be defined in a similar manner.

The assumption (1.4) implies that $\hat{\psi}$ is in the amalgam space $W\left(L^{\infty}, \ell^{2}\right)$.

From Theorem 2 follows that if $\psi$ satisfies (1.4), then $\left\{\tau_{y_{k}} \psi\right\}_{k \in \mathbb{Z}^{d}}$ is a 2 -Riesz basis of $V^{2}(\psi, Y)$ whenever $\left|k-y_{k}\right|_{\infty}<\frac{1}{4}$. See the remark after the proof of Theorem 2 in Section 4.

Exponential Riesz bases in $L^{2}(0,1)$ are completely understood and classified [20]. To the best of our knowledge, no such characterization exists for exponential bases on $L^{2}\left((0,1)^{d}\right)$ when $d>1$.

For our next theorem we consider $\psi$ in the Sobolev space $W_{0}^{1, p}\left(\mathbb{R}^{d}\right)$; we denote with $\partial_{j} \psi=\frac{\partial \psi}{\partial x_{j}}$ the partial derivative (in distribution sense) of $\psi$ and we let $\nabla \psi=\left(\partial_{1} \psi, \ldots, \partial_{d} \psi\right)$ be the gradient of $\psi$. Let $Y=\left\{y_{k}\right\}_{k \in \mathbb{Z}^{d}}$ and

$$
L=\sup _{k \in \mathbb{Z}^{d}}\left|y_{k}-k\right|_{2}<\infty \text {. }
$$

We prove the following

Theorem 3. Let $D=\left(a_{1}, b_{1}\right) \times \ldots \times\left(a_{d}, b_{d}\right)$ be a bounded rectangle in $\mathbb{R}^{d}$. Let $\psi \in W_{0}^{1, p}(D)$, with $1 \leq p<\infty$, and let $\left\{\tau_{k} \psi\right\}_{k \in \mathbb{Z}^{d}}$ be a $p$-Riesz basis of $V^{p}(\psi)$ with frame constants $0<A \leq B<\infty$. If

$$
C=L \sum_{j=1}^{d}\left(1+\left[b_{j}-a_{j}+L\right]\right)^{p-1}\left\|\partial_{j} \psi\right\|_{p}^{p}<A,
$$

the set $\left\{\tau_{y_{k}} \psi\right\}_{k \in \mathbb{Z}^{d}}$ is a $p$-Riesz basis of $V^{p}(\psi ; Y)$ with constants $B+C$ and $A-C$. 
The proofs of Theorems 2 and 3 are in Section 3.

Our Theorem 3 can be compared to [10, Theorem 3.5]. In this theorem it is assumed that $|\nabla(\psi)|$ is in the amalgam space $W\left(L^{\infty}, \ell^{1}\right)$, and that $\inf _{x \in[0,1)^{d}} \sum_{k \in \mathbb{Z}^{d}}|\nabla \psi(x+k)|_{2}>0$.

In the aforementioned theorem is proved that $\left\{\tau_{y_{k}} \psi\right\}_{k \in \mathbb{Z}^{d}}$ is a Riesz basis of $V^{2}(\psi ; Y)$ if $C^{\prime}=L^{2}(1+2 L)^{2 d}\|\nabla \psi\|_{W\left(L^{\infty}, \ell^{1}\right)}^{2}<A$. Generalizations to functions for which $|\nabla(\psi)|$ is in the amalgam space $W\left(L^{q}, \ell^{1}\right)$, with $q>d$ are also possible (see Remark 3.2 in [10]).

Our Theorem 3 reduces to [10, Theorem 3.5] when $p>d$ and $\psi$ has compact support. For example, when $\psi$ has support in $[0,1)^{d}$, the norm in $W\left(L^{p}, \ell^{1}\right)$ reduces to $\|\nabla \psi\|_{p}$. The constant $C$ in Theorem 3 may be smaller than $C^{\prime}$ defined above when the support of $\psi$ is small.

Theorem 3 does not apply when $\psi=$ rect or when $\psi$ is a step function; For $J \geq 1$, we let $\mathcal{S}_{J}=\left\{s(t)=\sum_{|j| \leq J} s_{j} \operatorname{rect}(t-j), s_{j} \in \mathbb{R}\right\}$. We let $p^{\prime}=\frac{p}{p-1}$ be the dual exponent of $p$. The following theorem is proved in Section 4.2 .

THEOREM 4. Assume that $g \in \mathcal{S}_{J}$ and that $\left\{\tau_{k} g\right\}_{k \in \mathbb{Z}}$ is a $p$-Riesz basis for $V^{p}(g)$, with frame constants $A$ and $B$. If

$$
2^{p} L J\|g\|_{p^{\prime}}^{p}<A,
$$

the sequence $\left\{\tau_{y_{k}} g\right\}_{k \in \mathbb{Z}}$ is a Riesz basis for $V^{p}(g ; Y)$.

Acknowledgement. We are grateful to the anonymous referee of this paper for her/his thorough reading of our manuscript and for providing suggestions that have improved the quality of our work.

We also wish to thank K. Hamm for providing a proof of the identity (1.3) for $p \neq 2$.

\section{Preliminaries}

2.1. Notation. We denote with $\langle f, g\rangle=\int_{\mathbb{R}^{d}} f(x) \bar{g}(x) d x$ and $\|f\|_{2}=$ $\sqrt{\langle f, f\rangle}$ the standard inner product and norm in $L^{2}\left(\mathbb{R}^{d}\right)$. For a given $p \in \mathbb{R}^{d}$ and $\delta>0$, we let $B(p, \delta)=\left\{x \in \mathbb{R}^{d}:|x-p|_{2}<\delta\right\}$.

We let $\operatorname{rect}(x)=\chi_{\left[-\frac{1}{2}, \frac{1}{2}\right)}(x)$ be the characteristic function of the interval $\left[-\frac{1}{2}, \frac{1}{2}\right)$ and $\beta^{s}=\operatorname{rect}^{(s+1)}(x)=\operatorname{rect} * \ldots * \operatorname{rect}(x)$ be the $s+1$-times iterated convolution of rect. The function $\beta^{s}(x)$, a piecewise polynomial function of degree $s$, is a $B$-spline of order $s$. See [27], where the B-splines were first introduced, and [21], 32] and the references cited there.

2.2. $p$ - Riesz bases. Recall that a Schauder basis in a separable Banach space $V$ is a linearly independent set $\mathcal{B}=\left\{v_{j}\right\}_{j \in \mathbb{Z}}$ such that: $\overline{\operatorname{span}(\mathcal{B})}=V$, and there exists a sequence of bounded linear functions $f_{j}: X \rightarrow \mathbb{C}$ (the functional coefficients of the basis) such that $x=\sum_{j} f_{j}(x) v_{j}$ for every $x \in V$. 
Following [6], 7] and [2, we say that $\mathcal{B}$ is a $p$-Riesz basis of $V$, with $1 \leq p<\infty$, if $\overline{\operatorname{Span}(\mathcal{B})}=V$, if every series $\sum_{n} a_{n} v_{n}$ converges in $V$ when $\vec{a}=\left(a_{n}\right)_{n \in \mathbb{Z}} \in \ell^{p}$ and if there exist constants $A, B>0$ such that, for every finite sequence of coefficients $\left\{d_{j}\right\}_{j \in \mathbb{Z}} \subset \mathbb{C}$, we have

$$
A\|\vec{d}\|_{\ell^{p}} \leq\left\|\sum_{j} d_{j} v_{j}\right\|_{p} \leq B\|\vec{d}\|_{\ell^{p}} .
$$

Every $p$-Riesz basis is a Schauder basis. As mentioned in the introduction, when $V=V^{p}(\psi)$ and $\psi$ has compact support, the functional coefficients of the basis $\left\{\tau_{k} \psi\right\}_{k \in \mathbb{Z}}$ of $V^{p}(\psi)$ can be written in terms of the dual functions of the basis.

The following results are well known (see e.g. [1, Prop. 1.1], [16], [24]).

Lemma 5. a) Let $\psi \in L_{0}^{p}\left(\mathbb{R}^{d}\right)$. The set $\mathcal{B}=\left\{\tau_{k} \psi\right\}_{k \in \mathbb{Z}^{d}}$ is a p-Riesz basis in $V^{p}(\psi)$ if and only if

$$
\sum_{m \in \mathbb{Z}^{d}}|\hat{\psi}(y+m)|^{2} \neq 0 \quad \text { for every } y \in\left[-\frac{1}{2}, \frac{1}{2}\right)^{d} .
$$

b) If $\psi \in W\left(L^{\infty}, \ell^{1}\right)$ is continuous and if $\mathcal{B}$ is Riesz basis in $V^{2}(\psi)$, then $\mathcal{B}$ is a $p$-Riesz basis in $V^{p}(\psi)$ for every $1 \leq p<\infty$.

Proof. For the convenience of the reader we prove that if $\psi \in L_{0}^{2}\left(\mathbb{R}^{d}\right)$, $\mathcal{B}$ is a Riesz basis of $V^{2}(\psi)$ with constants $0<A \leq B<\infty$ if and only if the following inequality holds for every $y \in Q=\left[-\frac{1}{2}, \frac{1}{2}\right)^{d}$.

$$
A \leq \sum_{m \in \mathbb{Z}^{d}}|\hat{\psi}(y+m)|^{2} \leq B
$$

We can verify (using e.g. the Poisson summation formula) that the function in (2.1) is continuous in $\bar{Q}$, and so (2.2) is equivalent to (2.1).

Let $\left\{c_{k}\right\}_{k \in \mathbb{Z}^{d}} \subset \mathbb{C}$ be a finite set of coefficients such that $\sum_{k}\left|c_{k}\right|^{2}=1$. The Fourier transform of $f=\sum_{k \in \mathbb{Z}^{d}} c_{k} \tau_{k} \psi$ is

$$
\hat{f}(y)=\hat{\psi}(y) \sum_{k \in \mathbb{Z}^{d}} c_{k} e^{2 \pi i y \cdot k}=\hat{\psi}(y) M(y) .
$$

and by Plancherel's theorem $\|f\|_{2}^{2}=\|\hat{f}\|_{2}^{2}=\sum_{m \in \mathbb{Z}^{d}} \int_{m+\left[-\frac{1}{2}, \frac{1}{2}\right)^{d}}|\hat{f}(y)|^{2} d y=$ $\sum_{m \in \mathbb{Z}^{d}} \int_{Q}|\hat{\psi}(y+m)|^{2}|M(y)|^{2} d y=\int_{Q}|M(y)|^{2} \sum_{m \in \mathbb{Z}^{d}}|\hat{\psi}(y+m)|^{2} d y$.

Let $g=\sum_{m \in \mathbb{Z}^{d}}|\hat{\psi}(y+m)|^{2}$; if (2.2) holds, from $\|f\|_{2}^{2}=$ $\int_{Q}|M(y)|^{2} g(y) d y$ and $\int_{Q}|M(y)|^{2} d y=\sum_{k}\left|c_{k}\right|^{2}=1$ follows that $A \leq\|f\|_{2}^{2} \leq$ $B$.

Conversely, from $A \sum_{k}\left|c_{k}\right|^{2} \leq\|f\|_{2}^{2} \leq B \sum_{k}\left|c_{k}\right|^{2}$ and the above considerations, follows that

$$
A\left\|M^{2}\right\|_{L^{1}(Q)} \leq \int_{Q}|M(y)|^{2} g(y) d y \leq B\left\|M^{2}\right\|_{L^{1}(Q)} .
$$


Every non-negative $h \in L^{1}(Q)$ can be written as $h=|M|^{2}$, with $M \in L^{2}(Q)$. The dual of $L^{1}(Q)$ is $L^{\infty}(Q)$ and so $\|g\|_{L^{\infty}(Q)}=\sup _{\|h\|_{L^{1}(Q)}=1} \int_{Q} f(y) g(y) d y$. From (2.3) follows that $A \leq\|g\|_{L^{\infty}(Q)} \leq B$ as required.

We will use the following Paley-Wiener type result.

Lemma 6. Let $X, Y \subset \mathbb{R}^{d}$ be countable and discrete. Suppose that $\left\{\tau_{x_{j}} \psi\right\}_{j \in \mathbb{Z}^{d}}$ is a $p$-Riesz basis of $V^{p}(\psi ; X)$ with constants $A \leq B$. If the inequality

$$
\left\|\sum_{j} a_{j}\left(\tau_{x_{j}} \psi-\tau_{y_{j}} \psi\right)\right\|_{p}^{p} \leq C \sum_{n}\left|a_{n}\right|^{p}
$$

holds for all finite sequences $\left\{a_{n}\right\}_{n \in \mathbb{Z}^{d}} \in \mathbb{C}$ with a constant $C<A$, the sequence $\left\{\tau_{y_{j}} \psi\right\}_{j \in \mathbb{Z}^{d}}$ is a $p$-Riesz basis of $V^{p}(\psi ; Y)$ with constants $B+C$ and $A-C$.

Proof. Assume that $\sum_{n}\left|a_{n}\right|^{p}=1$; we have:

$$
\left\|\sum_{j} a_{j} \tau_{y_{j}} \psi\right\|_{p} \leq\left\|\sum_{j} a_{j}\left(\tau_{x_{j}} \psi-\tau_{y_{j}} \psi\right)\right\|_{p}+\left\|\sum_{j} a_{j} \tau_{x_{j}} \psi\right\|_{p} \leq C+B
$$

and

$$
\left\|\sum_{j} a_{j} \tau_{y_{j}} \psi\right\|_{p} \geq\left\|\sum_{j} a_{j} \tau_{x_{j}} \psi\right\|_{p}-\left\|\sum_{j} a_{j}\left(\tau_{x_{j}} \psi-\tau_{y_{j}} \psi\right)\right\|_{p} \geq A-C .
$$

Proof of Theorem 1. Assume $p \in(1, \infty)$ and $\sum_{j}\left|a_{j}\right|^{p}=1$. Let $p^{\prime}=\frac{p}{p-1}$ is the dual exponent of $p$ and let $\left\{\delta_{j}\right\}_{j \in \mathbb{Z}^{d}}$ be a sequence of positive constants such that $\sum_{j}\left|\delta_{j}\right|^{p^{\prime}}<\infty$, We recall that, when $1<p<\infty$, a function $f \in L^{p}\left(\mathbb{R}^{d}\right)$ is in the Sobolev space $W^{1, p}\left(\mathbb{R}^{d}\right)$ if and only if there is a constant $c>0$ that depends on $f$ but not on $\delta$, such that

$$
\omega_{p}(\delta, f)=\sup _{|t|<\delta}\left\|\tau_{t} f-f\right\|_{p} \leq c \delta
$$

for every $\delta>0$. Furthermore, one can choose $c=\|\nabla f\|_{p}$. See e.g. Prop. 9.3 in 4]. By (2.4) and Hölder's inequality,

$$
\begin{gathered}
\left\|\sum_{j} a_{j}\left(\tau_{x_{j}} \psi-\tau_{y_{j}} \psi\right)\right\| \leq \sum_{j}\left|a_{j}\right|\left\|\tau_{x_{j}} \psi-\tau_{y_{j}} \psi\right\|_{p} \leq \sum_{j}\left|a_{j}\right| \delta_{j} \\
\leq c\left(\sum_{j}\left|a_{j}\right|^{p}\right)^{\frac{1}{p}}\left(\sum_{j}\left|\delta_{j}\right|^{p^{\prime}}\right)^{\frac{1}{p^{\prime}}}=c\left(\sum_{j}\left|\delta_{j}\right|^{p^{\prime}}\right)^{\frac{1}{p^{\prime}}} .
\end{gathered}
$$


We can chose the $\delta_{j}$ so small that $c\left(\sum_{j}\left|\delta_{j}\right|^{p^{\prime}}\right)^{\frac{1}{p^{\prime}}}<A$ and use Lemma 6 to complete the proof.

\section{Problem $1(p=2)$}

In this section we prove that Problem 1 can be solved when $p=2$ and $\hat{\psi}$ has compact support.

Theorem 7. Let $\psi \in L^{2}\left(\mathbb{R}^{d}\right)$. Assume that $\hat{\psi}$ has compact support and that there exist constants $c, C>0$ such that

$$
c \leq \inf _{x \in \mathbb{R}^{d}}|\hat{\psi}(x)| \leq \sup _{x \in \mathbb{R}^{d}}|\hat{\psi}(x)| \leq C .
$$

Let $\left\{\tau_{x_{j}} \psi\right\}_{j \in \mathbb{Z}^{d}}$ be a Riesz basis in $V^{2}(\psi, X)$. There exists $\delta>0$ such that if $Y=\left\{y_{j}\right\}_{j \in \mathbb{Z}^{d}} \subset \mathbb{R}^{d}$ satisfies $\sup _{j}\left|x_{j}-y_{j}\right|<\delta$, then also $\left\{\tau_{x_{j}} \psi\right\}_{j \in \mathbb{Z}^{d}}$ is a Riesz basis of $V^{2}(\psi)$.

Proof. Let $D=\operatorname{supp}(\hat{\psi})$. When $p=2$, Plancherel theorem implies that the set $\left\{\tau_{x_{j}} \psi\right\}_{j \in \mathbb{Z}^{d}}$ is a Riesz basis in $V^{2}(\psi)$ if and only if the set $\left\{e^{2 \pi i x_{j} \cdot x}\right\}_{j \in \mathbb{Z}^{d}}$ is a Riesz basis on $L^{2}\left(\mathbb{R}^{d}, \hat{\psi} d x\right)$. Our assumptions on $\hat{\psi}$ imply that the norm on $L^{2}\left(\mathbb{R}^{d}, \hat{\psi} d x\right)$ is equivalent to the norm on $L^{2}(D)$ and that $\left\{e^{2 \pi i x_{j} \cdot x}\right\}_{j \in \mathbb{Z}^{d}}$ is an exponential Riesz basis on $L^{2}(D)$. Exponential Riesz bases on bounded domains of $\mathbb{R}^{d}$ are stable under small perturbations (see [22] and also Section 2.3 in [18]); we can find $\delta>0$ such that, if $Y=\left\{y_{j}\right\}_{j \in \mathbb{Z}^{d}} \subset \mathbb{R}^{d}$ satisfies $\sup _{j}\left|x_{j}-y_{j}\right|<\delta$, then also $\left\{e^{2 \pi i y_{j} \cdot x}\right\}_{j \in \mathbb{Z}^{d}}$ is a Riesz basis on $L^{2}(D)$ and hence also in $L^{2}\left(\mathbb{R}^{d}, \hat{\psi} d x\right)$.

Example. Let $d=1$ and let $\psi=\operatorname{sinc}(x)=\frac{\sin (\pi x)}{\pi x}$; the Fourier transform of $\tau_{k} \psi(x)=\operatorname{sinc}(x-k)$ is $e^{2 \pi i k x} \operatorname{rect}(x)=e^{2 \pi i k x} \chi_{\left[-\frac{1}{2}, \frac{1}{2}\right)}(x)$, and so $V^{2}(\psi)$ is isometrically isomorphic to $\overline{\operatorname{Span}\left\{e^{2 \pi i j x} \operatorname{rect}(x)\right\}_{j \in \mathbb{Z}^{d}}}=L^{2}\left(-\frac{1}{2}, \frac{1}{2}\right)$. By Kadec's theorem $\left([\mathbf{1 7},[\mathbf{3 3}])\right.$ if $Y=\left\{y_{n}\right\}_{n \in \mathbb{Z}^{d}} \subset \mathbb{R}$ is $\operatorname{such}$ that $\sup _{n} \mid y_{n}-$ $n \mid \leq \delta<\frac{1}{4}$, the set $\left\{e^{2 \pi i y_{n} x}\right\}_{n \in \mathbb{Z}^{d}}$ is still a Riesz basis of $L^{2}\left(-\frac{1}{2}, \frac{1}{2}\right)$ and therefore, the set $\left\{\operatorname{sinc}\left(x-y_{n}\right)\right\}_{n \in \mathbb{Z}^{d}}$ is a Riesz basis for $V^{2}(\operatorname{sinc})$. Thus, $V^{2}(\sin c ; Y)=V^{2}(\sin c)$.

Things are not so clear when $p \neq 2$. For example, the trigonometric system $\mathcal{B}=\left\{e^{2 \pi i n x}\right\}_{n \in \mathbb{Z}^{d}}$ is a Schauder basis in $L^{p}\left(-\frac{1}{2}, \frac{1}{2}\right)$ when $1<p<\infty$, but it is not a $p$-Riesz basis and the previous example cannot be generalized in an obvious way. Stability results for the Schauder basis $\mathcal{B}$ in $L^{p}\left(-\frac{1}{2}, \frac{1}{2}\right)$ are proved in [25] and in [26].

\section{Problem 2}

In this section we prove Theorems 2 and 3 . Let $X=\left\{x_{n}\right\}_{n \in \mathbb{Z}^{d}}$ and $\mathcal{B}=\left\{e^{2 \pi i x \cdot x_{n}}\right\}_{n \in \mathbb{Z}^{d}}$. We first prove the following 
Lemma 8. Let $\psi \in L^{2}\left(\mathbb{R}^{d}\right) \cap L^{1}\left(\mathbb{R}^{d}\right)$ be as in (1.4); if $\mathcal{B}$ is a Riesz basis in $L^{2}\left([0,1)^{d}\right)$ with constants $A_{1}$ and $B_{1}$ then $\left\{\tau_{x_{n}} \psi\right\}$ is a Riesz basis of $\left.V^{2}(\psi, X)\right\}$ with constants $A=A_{1} c$ and $B=B_{1} C$.

Proof. For $k \in \mathbb{Z}^{d}$, we let $c_{k}=\inf _{x \in(0,1]^{d}}|\hat{\psi}(x+k)|^{2}$ and $C_{k}=$ $\sup _{x \in(0,1]^{d}}|\hat{\psi}(x+k)|^{2}$. Let $\left\{d_{j}\right\}$ be a finite set of complex coefficient such that $\sum_{j}\left|d_{j}\right|^{2}=1$. Since $\mathcal{B}$ is a Riesz basis in $L^{2}\left((0,1]^{d}\right)$, for every given $k \in \mathbb{Z}^{d}$ we have that

$$
A_{1} \leq\left\|\sum_{n} d_{n} e^{-2 \pi i x_{n} \cdot k} e^{2 \pi i x_{n} \cdot y}\right\|_{L^{2}\left((0,1]^{d}\right)}^{2} \leq B_{1} .
$$

From this inequality follows at once that

$$
c_{k} A_{1} \leq\left\|\sum_{n} d_{n} e^{-2 \pi i x_{n} \cdot k} e^{2 \pi i x_{n} \cdot y} \hat{\psi}(.-k)\right\|_{L^{2}\left((0,1]^{d}\right)}^{2} \leq C_{k} B_{1} .
$$

With $c=\sum_{k \in \mathbb{Z}^{d}} c_{k}$ and $C=\sum_{k \in \mathbb{Z}^{d}} C_{k}=\|\psi\|_{W\left(L^{\infty}, \ell^{2}\right)}^{2}$, we have

$$
A_{1} c \leq \sum_{k \in \mathbb{Z}^{d}}\left\|\sum_{n} d_{n} e^{2 \pi i x_{n} \cdot(.-k)} \hat{\psi}(.-k)\right\|_{L^{2}\left((0,1]^{d}\right)}^{2} \leq B_{1} C .
$$

In view of $\sum_{k \in \mathbb{Z}^{d}}\|g(.-k)\|_{L^{2}\left((0,1]^{d}\right)}=\|g\|_{2}$, we obtain

$$
A_{1} c \leq\left\|\sum_{n} d_{n} e^{2 \pi i x_{n} \cdot y} \hat{\psi}\right\|_{2}^{2} \leq B_{1} C .
$$

By Plancherel's theorem, the latter is equivalent to $A_{1} c \leq\left\|\sum_{n} d_{n} \tau_{x_{n}} \psi\right\|_{2} \leq$ $B_{1} C$ and so $\left\{\tau_{x_{k}} \psi\right\}_{k \in \mathbb{Z}^{d}}$ is a Riesz basis of $V^{2}(\psi, X)$, as required.

Proof of Theorem 2. Let $\mathcal{B}=\left\{e^{2 \pi i x \cdot x_{n}}\right\}_{n \in \mathbb{Z}^{d}}$ be a Riesz basis in $L^{2}\left([0,1)^{d}\right)$; it is proved in [22] (see also Section 2.3 in [18]) that we can find $\delta>0$ such that, if $Y=\left\{y_{j}\right\}_{j \in \mathbb{Z}^{d}} \subset \mathbb{R}^{d}$ satisfies $\sup _{j}\left|x_{j}-y_{j}\right|_{2}<\delta$, then also $\left\{e^{2 \pi i y_{j} \cdot x}\right\}_{j \in \mathbb{Z}^{d}}$ is a Riesz basis in $L^{2}\left([0,1)^{d}\right)$. By Lemma 8 , $\left\{\tau_{y_{n}} \psi\right\}$ is a Riesz basis of $V^{2}(\psi, Y)$.

Remark. When $Y=\left\{y_{k}\right\}_{k \in \mathbb{Z}^{d}}$ is such that $\sup _{k \in \mathbb{Z}^{d}}\left|k-y_{k}\right|_{\infty}<\frac{1}{4}$, by the multi-dimensional generalization of Kadec's theorem proved in [31] we have that $\left\{e^{2 \pi i y_{j} \cdot x}\right\}_{j \in \mathbb{Z}^{d}}$ is a Riesz basis in $L^{2}\left([0,1)^{d}\right)$ and by Lemma 8 , $\left\{\tau_{y_{n}} \psi\right\}_{n \in \mathbb{Z}^{d}}$ is a Riesz basis of $\left.V^{2}(\psi, Y)\right\}$.

4.1. Proof of Theorem 3 . In order to prove Theorem 3 we need some preliminary result: first, we prove the following 
Lemma 9. Let $(a, b) \subset \mathbb{R}$, with $a<b<\infty$, and let $1 \leq p<\infty$. Let $\psi \in L_{0}^{p}(a, b)$. For every finite set of coefficients $\left\{\alpha_{j}\right\} \subset \mathbb{C}$, we have that

$$
\left\|\sum_{k} \alpha_{k} \tau_{k} \psi\right\|_{p}^{p} \leq\|\psi\|_{p}^{p}([b-a]+1)^{p-1} \sum_{k}\left|\alpha_{k}\right|^{p}
$$

where [ ] denotes the integer part.

Proof. For simplicity we let $a=0$. When $b \leq 1$ the supports of the $\tau_{k} \psi$ 's are disjoint and so $\|f\|_{p}^{p}=\left\|\sum_{k} \alpha_{k} \tau_{k} \psi\right\|_{p}^{p}=\|\psi\|_{p}^{p} \sum_{k}\left|\alpha_{k}\right|^{p}$. When $b>1$ the supports of the $\tau_{k} \psi$ overlap, and there are at most $[b]+1$ of such supports that intersect at each point. By the elementary inequality $\left(x_{1}+\cdots+x_{m}\right)^{p} \leq m^{p-1}\left(x_{1}^{p}+\cdots+x_{m}^{p}\right)$ (which is valid when the $x_{j}$ are non-negative) we have that

$$
|f(t)|^{p}=\left|\sum_{k} a_{k} \tau_{k} \psi(t)\right|^{p} \leq([b]+1)^{p-1} \sum_{k}\left|a_{k}\right|^{p}\left|\tau_{k} \psi(t)\right|^{p}
$$

and so $\|f\|_{p}^{p} \leq([b]+1)^{p-1}\|\psi\|_{p}^{p} \sum_{k}\left|\alpha_{k}\right|^{p}$ as required.

Let $Y=\left\{y_{k}\right\}_{k \in \mathbb{Z}^{d}}$ be a discrete subset of $\mathbb{R}^{d}$. Assume that $L=$ $\sup _{k \in \mathbb{Z}^{d}}\left|y_{k}-k\right|_{2}<\infty$. We prove the following

Lemma 10. Let $D=\prod_{j=1}^{d}\left[a_{j}, b_{j}\right]$ and let $\psi \in W_{0}^{1, p}(D)$. Then, for every finite set of coefficients $\left\{\alpha_{j}\right\} \subset \mathbb{C}$ such that $\sum_{k}\left|\alpha_{k}\right|^{p}=1$, we have that

$$
\left\|\sum_{k} \alpha_{k}\left(\tau_{k} \psi-\tau_{y_{k}} \psi\right)\right\|_{p}^{p} \leq L \sum_{j=1}^{d}\left(1+\left[b_{j}-a_{j}+L\right]\right)^{p-1}\left\|\partial_{j} \psi\right\|_{p}^{p} .
$$

Proof. When $d=1$ and $D=(a, b)$, we prove that

$$
\left\|\sum_{k} \alpha_{k}\left(\tau_{k} \psi-\tau_{y_{k}} \psi\right)\right\|_{p}^{p} \leq L(1+[b-a+L])^{p-1}\left\|\psi^{\prime}\right\|_{p}^{p}
$$

where $\psi^{\prime}(t)$ denotes the distributional derivative of $\psi$. Assume first that $y_{k}>k$. Observing that $\psi\left(t+y_{k}\right)-\psi(t+k)=\int_{k}^{y_{k}} \psi^{\prime}(t+x) d x$ and that $\left|k-y_{k}\right| \leq L$, we have that

$$
\begin{aligned}
& \left\|\sum_{k} \alpha_{k}\left(\tau_{k} \psi-\tau_{y_{k}} \psi\right)\right\|_{p}^{p}=\left\|\sum_{k} \alpha_{k} \int_{t+k}^{t+y_{k}} \psi^{\prime}(x) d x\right\|_{p}^{p} \\
& \leq\left\|\sum_{k}\left|\alpha_{k}\right| \int_{t+k}^{t+k+L}\left|\psi^{\prime}(x)\right| d x\right\|_{p}^{p}=\left\|\sum_{k}\left|\alpha_{k}\right| \tau_{k} g\right\|_{p}^{p}
\end{aligned}
$$

where we have let $g(t)=\int_{t}^{t+L}\left|\psi^{\prime}(x)\right| d x$. It is easy to verify that $g(t)$ is supported in the interval $[a-L, b]$. Indeed, $\psi^{\prime}$ is supported in $[a, b]$ and so $g(t) \equiv 0$ whenever $t+[0, L] \cap[a, b]=\emptyset$. Thus, $g(t) \equiv 0$ when $t+L<a$ or $t>b$, or: $g(t) \equiv 0$ when $t \in \mathbb{R}-[a-L, b]$, as required. 
By Lemma 9

$$
\left\|\sum_{k} \alpha_{k}\left(\tau_{k} \psi-\tau_{y_{k}} \psi\right)\right\|_{p}^{p} \leq\left\|\sum_{k}\left|\alpha_{k}\right| \tau_{k} g\right\|_{p}^{p} \leq(1+[b-a+L])^{p-1}\|g\|_{p}^{p} .
$$

We apply a change of variables and Minkowsky's integral inequality; we gather

$$
\begin{gathered}
\|g\|_{p}=\left\|\int_{t}^{t+L}\left|\psi^{\prime}(x)\right| d x\right\|_{p}=\left\|\int_{0}^{L}\left|\psi^{\prime}(x+t)\right| d x\right\|_{p} \\
\leq L\left\|\psi^{\prime}\right\|_{p}
\end{gathered}
$$

which together with the inequality (4.3) concludes the proof of (4.2). When $y_{k}<k$ the proof if similar, but the function $g(t)$ defined above should be replaced by $g(t)=\int_{t}^{t-L}\left|\psi^{\prime}(x)\right| d x$, a function supported in the interval $[a, b+L]$.

When $d=2$ we can let $y_{k}=\left(y_{k, 1}, y_{k, 2}\right)$ and $k=\left(k_{1}, k_{2}\right)$ and write

$$
\begin{gathered}
\left\|\sum_{k} \alpha_{k}\left(\tau_{k} \psi-\tau_{y_{k}} \psi\right)\right\|_{p} \\
\leq\left\|\sum_{k} \alpha_{k}\left(\tau_{\left(k_{1}, k_{2}\right)} \psi-\tau_{\left(y_{k, 1}, k_{2}\right)} \psi\right)\right\|_{p}+\left\|\sum_{k} \alpha_{k}\left(\tau_{\left(y_{k, 1}, k_{2}\right)} \psi-\tau_{\left(y_{k, 1}, y_{k, 2}\right)} \psi\right)\right\|_{p} \\
=\left\|\sum_{k} \alpha_{k}\left(\tau_{k_{1}} \psi_{1}-\tau_{y_{k, 1}} \psi_{1}\right)\right\|_{p}+\left\|\sum_{k} \alpha_{k}\left(\tau_{k_{2}} \psi_{2}-\tau_{y_{k, 2}} \psi_{2}\right)\right\|_{p}
\end{gathered}
$$

where we have let $\psi_{1}=\tau_{\left(0, k_{2}\right)} \psi$ and $\psi_{2}=\tau_{\left(y_{k_{1}}, 0\right)} \psi$. The inequality (4.2), applied to $\psi_{1}$ and $\psi_{2}$, yields (4.1). The case $d>2$ is similar.

Proof of Theorem 3. Follows from Lemmas 6 and 10.

4.2. rect and step functions. Since Sobolev spaces $W^{1, p}(\mathbb{R})$ do not contain discontinuous functions, we cannot apply Theorem 3 when $\psi$ is a step function.

Let $\psi=$ rect; it is immediate to verify that, for every $1 \leq p<\infty$, the set $\left\{\tau_{j} \text { rect }\right\}_{j \in \mathbb{Z}}$ is a $p$-Riesz basis of $V^{p}$ (rect) with frame constants $A=B=1$. Throughout this section we let $Y=\left\{y_{k}\right\}_{k \in \mathbb{Z}} \subset \mathbb{R}$, with $L=\sup _{k \in \mathbb{Z}^{d}}\left|y_{k}-k\right|$ and we assume $1 \leq p<\infty$.

Lemma 11 below is an easy generalization of Lemma 10 in [8].

Lemma 11. Assume $0 \leq L<1$. For every finite set of coefficients $\left\{\alpha_{k}\right\}_{n \in \mathbb{Z}^{d}} \subset \mathbb{C}$ we have that

$$
\left\|\sum_{k} \alpha_{k}\left(\operatorname{rect}(t-k)-\operatorname{rect}\left(t-y_{k}\right)\right)\right\|_{p}^{p}<2^{p} L \sum_{k}\left|\alpha_{k}\right|^{p} .
$$


Proof. Assume $\sum_{k}\left|\alpha_{k}\right|^{p}=1$. Let

$$
f(t)=\sum_{k} \alpha_{k}\left(\operatorname{rect}(t-k)-\operatorname{rect}\left(t-y_{k}\right)\right)=\sum_{k} \alpha_{k} \chi_{I_{k}},
$$

where $I_{j}$ denotes the support of $\operatorname{rect}(t-j)-\operatorname{rect}\left(t-y_{j}\right)$. When $y_{j} \neq j, I_{j}$ is union of two intervals that we denote with $I_{j}^{+}$and $I_{j}^{-}$. When $y_{j}>j$, we let

$$
I_{j}^{-}=\left(j-\frac{1}{2}, y_{j}-\frac{1}{2}\right), \quad I_{j}^{+}=\left(j+\frac{1}{2}, y_{j}+\frac{1}{2}\right) .
$$

We use (improperly) the same notation to denote $I_{j}^{+}$and $I_{j}^{-}$also when $y_{j}<j$.

Since we have assumed $\left|y_{h}-h\right| \leq L<1$, for every given interval $J=I_{h}^{ \pm}$there is at most another interval $I_{k}^{ \pm}$that overlap with $J$; thus, for every $t \in \mathbb{R}$, the sum in (4.5) has at most 2 terms. By the elementary inequality $\left(x_{1}+\cdots+x_{m}\right)^{p} \leq m^{p-1}\left(x_{1}^{p}+\cdots+x_{m}^{p}\right)$ we have that $|f(t)|^{p} \leq 2^{p-1} \sum_{k}\left|\alpha_{k}\right|^{p} \chi_{I_{k}}(t)$, and $\|f\|_{p}^{p} \leq 2^{p-1} \sup _{k}\left|I_{k}\right|=2^{p-1}(2 L)=2^{p} L$ and the proof of the Lemma is concluded.

Lemma 11 and Lemma 6 yield the following

TheOREM 12. With the notation of Lemma 11, the set $\left\{\tau_{y_{k}} \text { rect }\right\}_{k \in \mathbb{Z}}$ is a $p$-Riesz basis in $V^{p}($ rect; $Y)$ if $2^{p} L<1$.

Corollary 13. Let $\psi_{0} \in L^{1}(\mathbb{R})$ and let $\psi=\operatorname{rect} * \psi_{0}$. Suppose that $\left\{\tau_{k} \psi\right\}_{k \in \mathbb{Z}}$ is a p-Riesz basis for $V^{p}(\psi)$. For every finite set of coefficients $\left\{\alpha_{k}\right\}_{n \in \mathbb{Z}} \subset \mathbb{C}$ with $\sum_{k}\left|\alpha_{k}\right|^{p}=1$, we have that

$$
\left\|\sum_{k} \alpha_{k}\left(\psi(t-k)-\psi\left(t-y_{k}\right)\right)\right\|_{p}^{p}<2^{p} L\left\|\psi_{0}\right\|_{1}^{p}
$$

and the set $\left\{\psi\left(t-y_{k}\right)\right\}_{k \in \mathbb{Z}}$ is a $p$-Riesz basis for for $V^{p}(\psi ; Y)$ whenever

$$
2^{p} L\left\|\psi_{0}\right\|_{1}^{p}<A
$$

Remark. If $\hat{\psi}_{0}(x) \neq 0$ for every $x \in \mathbb{R}$, then the set $\left\{\tau_{k} \psi\right\}_{k \in \mathbb{Z}}$ is a $p$-Riesz basis for $V^{p}(\psi)$. Indeed, $\sum_{m \in \mathbb{Z}}|\widehat{\operatorname{rect}}(y+m)|^{2}=\sum_{m \in \mathbb{Z}}|\operatorname{sinc}(x+k)|^{2} \neq$ 0 whenever $x \in\left[-\frac{1}{2}, \frac{1}{2}\right)$ and so also $\sum_{m \in \mathbb{Z}}|\hat{\psi}(x+k)|^{2}=\sum_{m \in \mathbb{Z}} \mid \hat{\psi}_{0}(x+$ $k)\left.\widehat{\operatorname{rect}}(x+k)\right|^{2} \neq 0$; by Lemma 5 the set $\left\{\tau_{k} \psi\right\}_{k \in \mathbb{Z}}$ is a $p$-Riesz basis for $V^{p}(\psi)$.

PROOF OF COROLlary 13, Let

$$
F(t)=\sum_{k} \alpha_{k}\left(\psi(t-k)-\psi\left(t-y_{k}\right)\right), \quad f(y)=\sum_{k \in \mathbb{Z}} \alpha_{k}\left(\operatorname{rect}(y-k)-\operatorname{rect}\left(y-x_{k}\right)\right.
$$


and we show that $\|F\|_{p}^{p} \leq 2^{p} L\left\|\psi_{0}\right\|_{1}^{p}$. We gather

$$
\begin{aligned}
F(t) & =\int_{-\infty}^{\infty} \psi_{0}(t-y) \sum_{k \in \mathbb{Z}} \alpha_{k}\left(\operatorname{rect}(y-k)-\operatorname{rect}\left(y-x_{k}\right)\right) d y \\
& =\psi_{0} * f(t) .
\end{aligned}
$$

Thus, by Young's inequality and Lemma 11,

$$
\|F\|_{p}^{p} \leq\left\|\psi_{0}\right\|_{1}^{p}\|f\|_{p}^{p} \leq 2^{p} L\left\|\psi_{0}\right\|_{1}^{p}
$$

and the proof of the corollary is concluded.

Let $\beta_{m}(x)=\operatorname{rect}^{(m+1)}$ be the B-spline of order $m>1$. We recall that $\beta_{m}$ is supported in the interval $\left[-\frac{m+1}{2}, \frac{m+1}{2}\right]$ and $\beta_{m}(x) \in W^{1, p}(\mathbb{R})$ whenever $m \geq 1$. It is easy to verify by induction on $m$ that $\left\|\beta^{m}\right\|_{p} \leq 1$ and $\left\|\beta_{m}^{\prime}\right\|_{p} \leq 2$. It is known that $\left\{\tau_{k} \beta_{m}\right\}_{k \in \mathbb{Z}}$ is a Riesz basis of $V^{2}\left(\beta_{m}\right)$ whose Riesz constants $A(m)$ and $B(m)$ are explicitly evaluated in [19. See also 28. By the observations after Lemma 5. $\left\{\tau_{k} \beta_{m}\right\}_{k \in \mathbb{Z}}$ is a $p$-Riesz basis of $V^{p}\left(\beta_{m}\right)$ with constants $A_{p}(m)>0$ and $B_{p}(m)<\infty$.

We prove the following

Corollary 14. Assume that $L<2^{-p} A_{p}(m)$. Then, the set $\left\{\tau_{y_{k}} \beta_{m}\right\}_{k \in \mathbb{Z}}$ is a p-Riesz basis of $V^{p}(\psi, Y)$.

Proof. We apply Corollary 13 with $\psi_{0}=\beta^{m-1}$.

Remark. We could have used Theorem 3 to prove Corollary 14, but we would have obtained a lower upper bound for $L$ (namely, $L<\frac{A_{p}(m)}{2(2+m)^{p-1}}$ ).

Proof of Theorem 4, Let $g(t)=\sum_{|j| \leq J} s_{j} \operatorname{rect}(t-j)$. Let $\left\{\alpha_{k}\right\}_{n \in \mathbb{Z}^{d}} \subset \mathbb{C}$ be a finite set of coefficients such that $\sum_{k}\left|\alpha_{k}\right|^{p}=1$. Let

$$
f(t)=\sum_{k} \alpha_{k}\left(g(t-k)-g\left(t-x_{k}\right)\right) .
$$

As in previous theorems, we find conditions on $L$ for which $\|f\|_{p}^{p}<A$. We have

$$
\begin{aligned}
f(t) & =\sum_{|j| \leq J} s_{j} \sum_{k} \alpha_{k}\left(\operatorname{rect}(t-j-k)-\operatorname{rect}\left(t-j-x_{k}\right)\right) \\
& =\sum_{|j| \leq J} s_{j} f_{j}(t) .
\end{aligned}
$$


By Minkowski and Hölder inequalities, and noting that $\sum_{|j| \leq J}\left|s_{j}\right|^{q}=\|g\|_{q}^{q}$, it follows that

$$
\begin{aligned}
\|f\|_{p} \leq \sum_{|j| \leq J}\left|s_{j}\right|\left\|f_{j}\right\|_{p} & \leq\left(\sum_{|j| \leq J}\left|s_{j}\right|^{p^{\prime}}\right)^{\frac{1}{p^{\prime}}}\left(\sum_{|j| \leq J}\left\|f_{j}\right\|_{p}^{p}\right)^{\frac{1}{p}} \\
& =\|g\|_{p^{\prime}}\left(\sum_{|j| \leq J}\left\|f_{j}\right\|_{p}^{p}\right)^{\frac{1}{p}},
\end{aligned}
$$

With the change of variables $t-j=t^{\prime}$ in the integral below, we obtain

$$
\begin{aligned}
& \int_{\mathbb{R}}\left|f_{j}(t)\right|^{p} d t=\int_{\mathbb{R}}\left|\sum_{k} \alpha_{k}\left(\operatorname{rect}(t-j-k)-\operatorname{rect}\left(t-j-x_{k}\right)\right)\right|^{p} d t \\
&=\int_{\mathbb{R}}\left|\sum_{k} \alpha_{k}\left(\operatorname{rect}\left(t^{\prime}-k\right)-\operatorname{rect}\left(t^{\prime}-x_{k}\right)\right)\right|^{p} d t^{\prime} \\
&=\| \sum_{k} \alpha_{k}\left(\operatorname{rect}(t-k)-\operatorname{rect}\left(t-y_{k}\right) \|_{p}^{p} .\right.
\end{aligned}
$$

From Lemma 11, follows that the integral above is $\leq 2^{p} L$. We gather: $\|f\|_{p}^{p} \leq 2^{p} L J\|g\|_{p^{\prime}}^{p}$. By assumption $2^{p} L J\|g\|_{p^{\prime}}^{p}<\bar{A}$, and by Lemma 6 Theorem 4 follows.

\section{Remarks and open problems}

We have discussed Problem 1 when $p=2$ and the Fourier transform of the window function $\psi$ has compact support. When $\psi$ is not band-limited, Plancherel's theorem implies that the set $\left\{\tau_{x_{j}} \psi\right\}_{j \in \mathbb{Z}^{d}}$ is a Riesz basis in $V^{2}(\psi, X)$ if and only if the set $\mathcal{V}=\left\{e^{2 \pi i x_{j} \cdot x}|\hat{\psi}|\right\}_{j \in \mathbb{Z}^{d}}$ is a Riesz sequence in $L^{2}\left(\mathbb{R}^{d}\right)$, and hence a Riesz basis in $V=\overline{\operatorname{Span}(\mathcal{V}))}$. By a theorem of Krein-Milman-Rutman (see e.g. [33, Theorem 11]) for every $j \in \mathbb{Z}^{d}$ there exists $\epsilon_{j}>0$ such that every set of functions $\left\{g_{j}\right\}_{j \in \mathbb{Z}^{d}} \subset V$ is a Riesz basis of $V$ whenever $\left\|g_{j}-e^{\pi i x_{j} \cdot x} \mid \hat{\psi}\right\|_{2}<\epsilon_{j}$. We can find $\delta_{j}>0$ such that $\left\|\left(e^{\pi i x_{j} \cdot x}-e^{\pi i y_{j} \cdot x}\right) \hat{\psi}\right\|_{2}<\epsilon_{j}$ whenever $\left|x_{j}-y_{j}\right|_{2}<\delta_{j}$, but we do not know whether the $\delta_{j}$ 's have a lower bound or not.

For functions $\psi$ in $L^{p}\left(\mathbb{R}^{d}\right)$ for every $p \in[1, \infty)$ it would be interesting to prove conditions that would ensure that a $q$-Riesz basis in $V^{q}(\psi, X)$ for some $q$ is automatically a $p$-Riesz basis in $V^{p}(\psi, X)$ for all $p$. Lemma 5 (b) shows that, for certain $\psi$, if the set $\left\{\tau_{k} \psi\right\}_{k \in \mathbb{Z}^{d}}$ is a 2-Riesz basis of $V^{2}(\psi)$, it is also a p-Riesz in $V^{p}(\psi)$ but the method of proof of this result does not generalize well to other sets of translations. Results in [3] and [29] may help generalize Lemma 5 , 
It would also be interesting to define and investigate p-Riesz bases in quasi-shift invariant spaces $V^{p}(\psi, X)$ when $0<p<1$. Wavelet in $L^{p}$ with $0<p<1$ have been considered in [12]. We feel that the results contained in Section 3 of [12] may help the understanding of $V^{p}(\psi, X)$ when $0<p<1$.

\section{References}

[1] A. Aldroubi, Q. Sun, Connection between $p$-frames and $p$-Riesz bases in locally finite SIS of $L^{p}(\mathbb{R})$ Proceedings of SPIE - The International Society for Optical Engineering, February 1970

[2] A. Aldroubi, Q. Sun, W. Tang, p-Frames and shift invariant subspaces ofL ${ }^{p}$, J. Fourier Anal. Appl. 7 (2001) 1-21.

[3] A. Aldroubi, A. Baskakov, I. Krishtal, Slanted matrices, Banach frames, and sampling, J. Funct. Anal. 255 (2008) 1667-1691.

[4] H. Brezis, Functional Analysis, Sobolev Spaces and Partial Differential Equations, Springer Verlag 2011.

[5] A. Ben-Artzi, A. Ron, On the Integer Translates of a Compactly Supported Function: Dual Bases and Linear Projectors, SIAM J. Math. Anal., 21(6), 15501562.

[6] P. Casazza, O. Christensen, D.T. Stoeva, Frame expansions in separable Banach spaces, J. Math. Anal. Appl. 307 (2005) 710-723.

[7] O. Christensen, D.T. Stoeva, p-Frames in separable Banach spaces, Adv. Comput. Math. 18 (2003) 117-126.

[8] L. De Carli, P. Vellucci, Stability theorems for the n-order hold model, arXiv:1605.01706 (2016) (submitted)

[9] H. Feichtinger, Spline-type spaces in Gabor analysis. Wavelet analysis (Hong Kong, 2001), 100122, Ser. Anal., 1, World Sci. Publ., River Edge, NJ, 2002.

[10] H. Feichtinger, U. Molter, J.L.Romero, Perturbation techniques in irregular splinetype spaces, World Scientific Publishing Co. Inc., Int. J. Wavelets Multiresolut. Inf. Process, 6 (2) (2008) 249277.

[11] H. Feichtinger, H.; D.M. Onchi, Constructive reconstruction from irregular sampling in multi-window spline-type spaces. Progress in analysis and its applications, 257265, World Sci. Publ., Hackensack, NJ, 2010.

[12] G. Garrigos; R. Hochmuth; A. Tabacco, Wavelet characterizations for anisotropic Besov spaces with $0<p<1$ Proc. Edinburgh Math. Soc. 47 (2004) 573-59.

[13] K. Gröchenig, J. Stöckler, Gabor Frames and Totally Positive Functions, Duke Math. J. 162(6) (2013), 1003-1031.

[14] K. Hamm, J. Ledford, On the structure and interpolation properties of quasi-invariant shift spaces, arxiv:1703.01533 (2017)

[15] C. Heil, A basis theory primer, Appl. Num. Harm. Analysis, Birkhäuser 2011.

[16] R.Q. Jia, C. A. Micchelli, Using the refinement equation for the construction of prewavelets II: power of two, In "Curve and Surface" (P. J. Laurent, A. Le Mehaute and L. L. Schumaker eds.), Academic Press, New York 1991, pp. 209-246.

[17] M.I. Kadec, The exact value of the Paley-Wiener constant, Soviet Math. Dokl., 5 (1964), 559-561.

[18] G. Kozma, S. Nitzan, Combining Riesz bases, Inv. Math. 199 (1) (2015), , pp 267-285

[19] E.V. Mischenko, Determination of Riesz bounds for the spline basis with the help of trigonometric polynomials. Sib. Math. J. 51(4), 660-666 (2010)

[20] B. Pavlov, Basicity of an exponential system and Muckenhoupt's condition, Soviet Math. Dokl. 20 (1979) 655-659.

[21] Prautzsch, H., Boehm, W., Paluszny, M., Bezier and B-Spline Techniques, Springer Science and Business Media (2002).

[22] R. Paley and N. Wiener, Fourier transforms in the complex domain. Amer. Math. Soc. Colloquium Publications, vol. 19; Amer. Math. Soc., New York, 1934. 
[23] J.L. Romero, Explicit localization estimates for spline-type spaces. Sampl. Theory Signal Image Process. 8 (2009), no. 3, 249-259.

[24] A. Ron, A necessary and sufficient condition for the linear independence of the integer translates of a compactly supported distribution, Constructive Approximation 5 (1), 297-308.

[25] A. M. Sedletskii, Izv. Vyssh. Uchebn. Zaved., Mat., No. 7, 85-91 (1973).

[26] A. M. Sedletskii, Equivalence of the trigonometric system and its perturbations in $L^{p}(\pi, \pi)$, Doklady Mathematics. Vol. 94. No. 1. Pleiades Publishing, 2016.

[27] I. J. Schönberg, Cardinal interpolation and spline functions. J. Approx. teory 2 (2) (1969), pp. 167-206.

[28] A. Antony Selvan, R. Radha, Sampling and Reconstruction in Shift Invariant Spaces of B-Spline Functions Acta Appl. Math. DOI 10.1007/s10440-016-0053-6 (2016)

[29] C. E. Shin, Q. Sun, Stability of localized operators, J. Funct. Anal. 256 (2009) 24172439.

[30] Q. Sun, Stability of the Shifts of Global Supported Distributions J.Math. Analysis and Appl. 261 (1)(2001) 113-125.

[31] W. Sun, X. Zhou, On the stability of multivariate trigonometric systems. J. Math Anal. Appl. 235 (1999), 159-167.

[32] M. Unser, A. Aldroubi, M. Eden, B-spline signal processing. I. Theory, Signal Processing, IEEE Transactions on $41(2), 821-833$.

[33] R. M. Young, An introduction to nonharmonic Fourier series, Academic Press, 2001

Laura De Carli: Florida International Univ., Univ. Park, Miami (FL)

E-mail address: decarlil@fiu.edu

Pierluigi Vellucci: Dept. of Economics, Roma Tre University, via Silvio

D'Amico 77, 00145 Rome, Italy.

E-mail address: pierluigi.vellucci@uniroma3.it 